\title{
ORBITAL PERIOD VARIATION STUDY OF THE ALGOL ECLIPSING BINARY DI PEGASI
}

\author{
M. A. Hanna \& S. M. Amin \\ National Research Institute of Astronomy and Geophysics (NRIAG), Helwan, Cairo 11421, Egypt \\ (Received May 7, 2013; Accepted May 31, 2013)
}

\begin{abstract}
We discuss the orbital period changes of the Algol semi-detached eclipsing binary DI Peg by constructing the $(O-C)$ residual diagram via using all the available precise minima times. We conclude that the period variation can be explained by a sine-like variation due to the presence of a third body orbiting the binary, together with a long-term orbital period increase $(d P / d t=0.17 \mathrm{sec} /$ century $)$ that can be interpreted to be due to mass transfer from the evolved secondary component (of rate $1.52 \times 10^{-8} \mathrm{M}_{\odot} / \mathrm{yr}$ ) to the primary one. The detected low-mass third body $\left(M_{3} \min .=0.22 \pm 0.0006 \mathrm{M}_{\odot}\right)$ is responsible for a periodic variation of about 55 years light time effect. We have determined the orbital parameters of the third component which show a considerable eccentricity $e_{3}=0.77 \pm 0.07$ together with a longitude of periastron $\omega_{3}=300^{\circ} \pm 10^{\circ}$.
\end{abstract}

Key words : binaries: eclipsing — binaries: close — binaries: Algol — stars: triple — individual: DI Peg

\section{INTRODUCTION}

DI Peg (BD+14 5006, HD 220619, HIP 116167, $\alpha_{2000}$ $=23^{\mathrm{h}} 32^{\mathrm{m}} 14^{\mathrm{s}} .66924, \delta_{2000}=+14^{\circ} 58^{\prime} 08^{\prime \prime} .7408, \mathrm{sp}$. F0IVn, $\operatorname{mag}_{\mathrm{v}}=9.51$ according to Simbad database) is a relatively short period $\left(P=0^{\mathrm{d}} .7118\right)$ eclipsing binary, that has been discovered as an Algol type variable by Morgenroth (1934).

The system was observed by Jensch (1934) who published a photographic light curve. Subsequently, photoelectric light curves were observed by several authors; Kruszewski in 1962 (V filter), Chou \& Kitamura (1968), and Binnendijk (1973) in B\&V filters, and Chaubey (1982) in U, B\&V filters.

High dispersion spectroscopic observations were conducted by Lu (1992) who presented the radial-velocity measurements of DI Peg and determined a K4 spectral type for the secondary component. He established the triplicity of the system confirming the earlier finding of Rucinski (1967) who suggested the presence of a third source of light based on an analysis of light curves contributing $24 \%$ of the total light of the system. Lu (1992) determined the radial velocity of the third component $(+40.2 \pm 0.3 \mathrm{~km} / \mathrm{s})$, and also carriedout a detailed analysis of the published photoelectric light curves with the Wilson-Devinney code (Wilson, 1979) setting the third light $l_{3}$ as a free parameter. He obtained the following absolute dimensions of DI Peg as: $A=4.14 \pm 0.05 \mathrm{R}_{\odot} ; R_{1}=1.41 \pm 0.03 \mathrm{R}_{\odot}$; $R_{2}=1.37 \pm 0.03 \mathrm{R}_{\odot} ; M_{1}=1.18 \pm 0.03 \mathrm{M}_{\odot} ;$ and $M_{2}=0.70 \pm 0.02 \mathrm{M}_{\odot}$.

The aim of this paper is to study the changes in the orbital period of this interesting system and to re- determine the third body orbital parameters using the photoelectric and CCD minima times obtained over the last two decades. All the available data covering about 84 years from 1928 to 2012 .

\section{PERIOD VARIATION STUDY}

\subsection{Light Elements}

The light elements of DI Peg was first obtained by Jensch (1934):

$$
\text { HJD(Min.I) }=2425644.315+0^{\mathrm{d}} .711811 E .(1)
$$

Later, several authors observed minima times visually (v), photographically (pg), photoelectrically (pe \& CCD). They obtained different light elements that are listed in Table 1. We used Kreiner's (2004) light elements (see Table 1 ) to calculate the residual values of the $(O-C)$ diagram. For minima time detection, we used the last set of pe and CCD minima times of Table 7 (43 minima) starting from the Julian date 2452567.3312 to JD. 2456163.4447 to obtain the light elements:

$$
\text { HJD(Min.I) }=2425918.21913+0^{\mathrm{d}} .71181981 E .
$$

with standard deviation $S D=0.0013$ and regression $r$ $=0.9643$.

To study the period variation of DI Peg, one must first know the evolutionary status of the system through the spectroscopic and photoelectric light curves analysis of the previous studies. Second, one can construct 
Table 1.

\begin{tabular}{|c|c|c|c|c|}
\hline JD. +240000 & Period $(d)$ & Quad. term & Periodic term & Reference \\
\hline 25644.3150 & 0.71181100 & & & Jensch (1934). \\
\hline 32441.4410 & 0.71181400 & & & Jensch \& Szafraniec (1951), SAC 22. \\
\hline 32441.4410 & 0.71181988 & & & Gaposchkin (1952). \\
\hline 32441.4350 & 0.71181840 & & & Rucinski (1967). \\
\hline 37522.3946 & 0.71181750 & & & Chaubey (1982). \\
\hline 37983.6528 & 0.71181880 & & & Chou \& Kitamura (1968). \\
\hline 40114.8356 & 0.71181510 & & & Binnendijk (1973). \\
\hline 25918.346 & 0.71181730 & & $a \sin [b(E+c)]^{\dagger}$ & von Ahnert (1974). \\
\hline 42411.86193 & 0.71181612 & & & Mallama (1980). \\
\hline 32441.4470 & 0.71181768 & $-0.194 \times 10^{-9}$ & $a \sin (b E+c)^{\dagger \dagger}$ & Rafert (1982). \\
\hline 25918.3597 & 0.71181663 & & & $\mathrm{Lu}(1992)$ \\
\hline 45196.488 & 0.71181680 & & & Kholopov et al., (1998). \\
\hline 25918.261 & 0.71181641 & & & Kreiner (2004). \\
\hline 25918.23085 & 0.71181951 & & & Present Work ${ }^{\ddagger}$ \\
\hline 25918.36771 & 0.71181545 & $1.901 \times 10^{-11}$ & & Present Work ${ }^{\ddagger \ddagger}$ \\
\hline
\end{tabular}

$\dagger a=0^{d} .0055, b=0^{\circ} .0221765$ and $c=3000$.

计 $a=0^{d} .0107, b=0^{\circ} .0001580$ and $c=6495$.

$\ddagger$ Light elements obtained by using Pe and CCD minima starting from JD 24 52567.3312.

†† Quad. light elements obtained by using all Pe and CCD minima only.

the $O-C$ diagram in order to:

(1) discuss the effect of mass transfer and/or lost from the system,

(2) to deduce the light time effect (LITE) due to the presence of a third body that has been already detected spectroscopically by $\mathrm{Lu}$ (1992), then calculating the third body orbital parameters, and

(3) to discuss the possibility of the presence of starspots due to magnetic activity.

\subsection{Previous Light Curve Studies}

Rucinski (1967) used the unpublished light curve of Kruszewski's 1961 observations in the yellow band to obtain the geometrical elements of DI Peg. He introduced an additional (third) source contributing $24 \%$ of the light and identified a secondary minimum placed exactly at a phase of 0.5 (i.e., $e=0$ ), and obtained a F4IV spectral type for the primary component. He also suggested a semi-detached configuration of the system with two evolved components from the main sequence in which the fainter filled its Roche lobe.

Chou \& Kitamura (1968) observed the system photoelectrically with B \& V filters. They suggested a semi-detached Algol type configuration for DI Peg with sp. type $\mathrm{K} 0+\mathrm{K} 2$.

Binnendijk (1973) has also observed the system with B \& V filters, and concluded that the extra light hypothesis of Rucinski (1967) is in conflict with his observations, but he agreed with him in the semi-detached property of the system.

Using Wood's (1972) model, Mardirossian et al. (1980) analyzed Binnendijk's (1973) two-color photo- metric observations and the photoelectric light curve obtained by Kruszewski and published by Rucinski (1967). Their analysis showed a cooler under-massive component which is a typical property of post-main sequence mass-exchange of Algol-type binaries.

Since the spectroscopic mass ratio q was unknown, Rucinski (1967) used $q=0.3$, while Mardirossian et al. (1980) used $q=0.6$ during their light curves analysis. Both studies agreed in their conclusion about the semidetached configuration of DI Peg, but disagreed in the values for the orbital elements. They also disagreed about the necessity for the third light source.

Mardirossian et al. (1980) deduced the radii and the luminosities $R_{1}=1.4 \mathrm{R}_{\odot}, R_{2}=1.1 \mathrm{R}_{\odot}, \log \left(L_{1} / \mathrm{L}_{\odot}\right)=$ $0.50, \log \left(L_{2} / \mathrm{L}_{\odot}\right)=-0.17$ under the uncertainty of their assumption that $M_{1}=1.4 \mathrm{M}_{\odot}$.

Chaubey (1982) has observed DI Peg photoelectrically in the three UBV filters. The observed light curves showed luminosity at phase $0^{\mathrm{d}} .25$ to be greater than the luminosity at phase $0^{\mathrm{d}} .75$ in all the UBV filters. He argued that this phenomenon was either due to gas stream absorption or to electron scattering present in the system as noted by Piotrowski et al. (1974) in similar systems such as e.g., U Cep, U CrB, SW Cyg and S Equ. Another phenomenon that was seen in the light curves is that the shoulders of the primary minimum are depressed, which may be explained by a disc of circumstellar material surrounding the hotter component. The reduction in the light is then due to the eclipse of the disk by the subgiant component before and after the primary component is eclipsed (Chaubey, 1982). His orbital solution also suggested the semi- 
detached configuration. Using the minima times available, Chaubey constructed the $O-C$ diagram and observed an inversion to the increase trend after 1969. He explained this change in the period with Biermann and Hall's (1973) model which incorporates mass transfer in the system.

The period variation of DI Peg have been observed by several authors (e.g., Rusinski 1967, Binnendijk 1973, Chaubey 1982, Kennedy 1982, Lu 1992 and Vinkó 1992), but few studies of these changes were performed in the literatures (e.g., Chou \& Kitamura 1968, Lu 1992, and Vinkó 1992).

\subsection{The $O-C$ Diagram}

Kennedy (1982) observed four minima and combined them with other published minima. He constructed the $O-C$ diagram from data covering about 50 years. The diagram indicated two significant changes in the orbital period. A sudden period increase of $2^{\mathrm{d}} .4 \times 10^{-6}$ around 1946 and an almost identical sudden period decrease of $2^{\mathrm{d}} .2 \times 10^{-6}$ around 1969 (Kennedy 1982).

Besides the spectroscopic detection of a third body by $\mathrm{Lu}(1992)$, he established the $O-C$ diagram showing its sinusoid shape structure, but he did not use it to estimate the third body orbital parameters. However, the only study for determining the orbital parameter values of the third body was carried out by Vinkó (1992) (see Table 2). He suggested a third body orbiting the binary in 22 years, and reported that his derived orbital parameters are uncertain due to the shortness of the observed time interval.

Currently, more precise pe and CCD minima times are available since the last study of Vinkó in 1992. Hence, we aim to revisit the period variability of this interesting late type sd-Algol in order to calculate its third body orbital parameters as well as to discuss the other possible mechanisms that may affect the change in the period of DI Peg.

The period variability of DI Peg has been studied by means of an $O-C$ diagram analysis. We have used the following data reduction procedure. All the available times of mid eclipse have been gathered and examined carefully. A mean value of the observed time of minima, in the same epoch, for different filter bands, e.g., U, B and $\mathrm{V}$ has been used. The precise photoelectric and CCD times of minimum are used in our computations with weight 10 . The three CCD times of minimum at JD $2452542.7862,2452572.6843$ and 2452573.0329 $(E=37403.5,37445.5$ and 37446$)$ obtained by Karska and Maciejewski (2003) in addition to the pe secondary minimum 2454070.3254 ( $E=39549.5)$ by Senavci et al. (2007) are excluded due to their very large deviations from the general trend of the $O-C$ diagram. They are typed in Table 4 in italics. The earlier pg data before the JD 2437196.391 are not used due to their large scatter, while the others are used with weight 3 . Some visual minima are used with weight 1 to fill gaps in the diagram and the others are not used to minimize the

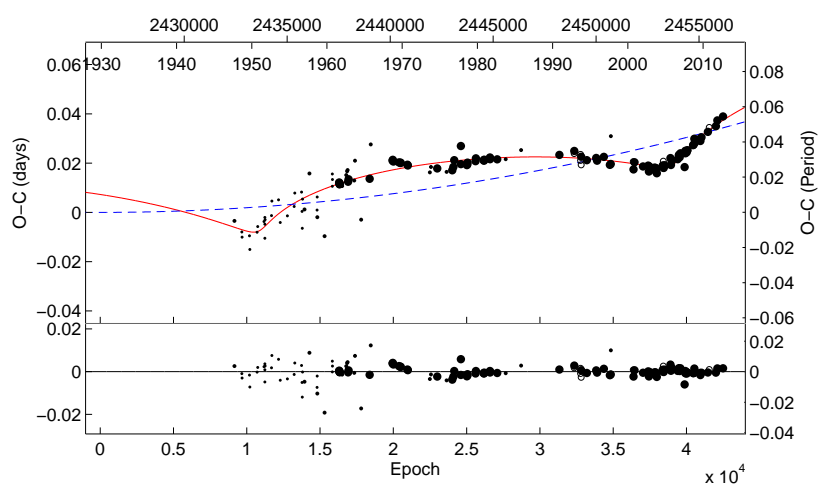

Fig. 1. - Raw $O-C$ diagram for DI Peg based on Kreiner's ephemeris (upper panel). The individual observations are shown as dots (primary) and open circles (secondary), the size is proportional to the statistical weight assigned to the individual minima (visual - 1; photographic - 3; photoelectric \& CCD measurement - 10). The lower panel represents the residuals after the subtraction of the solution.

contamination of the data set. However, all the minima times used have been presented in Table 3. The unused data are listed in Table 4, for completeness. A total of 145 (70 pe, 28 CCD, $18 \mathrm{pg}$ and $29 \mathrm{v}$ ) times of minima were used in our analysis. The $O-C$ values have been computed using the linear ephemeris of Kreiner (2004) (Table 1 ) and the resulting $O-C$ curve is displayed in the upper panel of Fig. 1.

\subsubsection{Mass Transfer and Light Time Effect}

On analyzing the $O-C$ diagram we used the standard approach (see, e.g., Mayer 1990 and Awadalla et al. 2004) assuming that the time of minima follow a quadratic ephemeris and are modulated by light time effect (LITE: see, e.g., Irwin 1959). The time of mid eclipse can be computed as follows:

$$
\begin{aligned}
\text { Min.I }= & J D_{0}+P \cdot E+Q \cdot E^{2}+\frac{a_{12} \sin \mathrm{i}}{c} \times \\
& {\left[\frac{1-e_{3}^{2}}{1+e_{3} \cos \nu} \sin \left(\nu+\omega_{3}\right)+\mathrm{e}_{3} \sin \omega\right], }
\end{aligned}
$$

where $e_{3}, \omega_{3}, \nu, a_{12} \sin i$ and c are the eccentricity, longitude of the periastron, true anomaly of the binary orbit around the center of mass of the triple system, projected semi-major axis, and the speed of light, respectively. We have used the computer programme written by Zasche et al. (2009). The quadratic ephemeris of the minima is represented by the first three terms of Eq. 3, and represented as the dashed line in Fig. 1, while the solid line fit represents the light time effect. The lower panel shows the residuals after the subtraction of the solution. 
Table 2.

\begin{tabular}{|c|c|c|c|}
\hline & & Vinkó (1992) & Present Work \\
\hline$P_{3}($ period $)$ & [yr.] & 22.09 & $54.98 \pm 0.76$ \\
\hline$A$ (semi-amplit.) & [day] & - & $0.0101 \pm 0.0068$ \\
\hline$e_{3}$ (eccentricity) & & $0.66 \pm 0.2$ & $0.77 \pm 0.07$ \\
\hline$\omega_{3}$ long. preias. pass. & {$[\mathrm{rad}]$} & $2.5 \pm 0.4$ & $5.25 \pm 0.18$ \\
\hline Time of periastron passage $T_{0}$ & [HJD] & $2440612 \pm 400$ & $2433702.022 \pm 344.419$ \\
\hline$a_{12} \sin i$ (projection of semi-major axis) & {$[\mathrm{AU}]$} & $0.77 \pm 0.21$ & $1.91 \pm 0.13$ \\
\hline$f\left(M_{3}\right)$ & {$\left[\mathrm{M}_{\odot}\right]$} & $0.001 \pm 0.001$ & $0.0023 \pm 0.000002$ \\
\hline$M_{3 i=90^{\circ}}$ & {$\left[\mathrm{M}_{\odot}\right]$} & - & $0.22 \pm 0.00006$ \\
\hline$i=60^{\circ}$ & & - & $0.26 \pm 0.00007$ \\
\hline$i=30^{\circ}$ & & - & $0.48 \pm 0.00015$ \\
\hline$a_{3}$ angular distance of $3^{r d}$ component & {$[\mathrm{mas}]$} & - & 96.421 \\
\hline$J D_{0}$ & [HJD] & - & $2425918.36771 \pm 0.00136$ \\
\hline Pinary & [day] & - & $0.711815447 \pm\left(0.5 \times 10^{-7}\right)$ \\
\hline$Q \quad\left(\times 10^{-11}\right)$ & [day] & - & $1.901 \pm 0.00071$ \\
\hline Sum of the square residuals $\sum(O-C)^{2}$ & {$\left[\right.$ days $\left.^{2}\right]$} & - & 0.0071 \\
\hline
\end{tabular}

Many semi-detached Algol binary systems exhibit increase in orbital periods during their evolution, while others show decrease. Generally, it has been thought that conservative mass transfer in Algol binaries causes their orbits to be wider because mass transfers from the evolved less massive secondary star to its more massive main sequence companion (e.g., AK Ser in Qian 2000; W Del in Hanna 2006; CL Aur in Wolf et al. 2007; RR Dra and TZ Eri in Zasche, et al. 2008). In case of Algols that show orbital period decrease (e.g., RZ Dra in Kreiner et al. 1994; UX Her in Tremko et al. 2004; AT Peg in Hanna 2012), authors (e.g., Kreiner et al. 1994; Pribulla, 1998) have attributed such decrease to mass loss from the system (non-conservative) via the lagrangian point $L_{2}$.

As one can see from the quadratic term in Eq. 3 (see Table 2), there is a long-term evolution of the orbital period represented by the dashed line in Fig. 1. It may be identified as a period increase caused by slow mass transfer rate $\mathrm{dP} / \mathrm{dt}(=0.17 \mathrm{sec} /$ century $)$ from the evolved less massive secondary component to its more massive companion star. The rate of mass transfer in the conservative case can be estimated by using the formula derived by Kreiner and Ziolkowski (1978):

$$
\dot{M}=243.5 \frac{Q}{P^{2}} \frac{M_{1} M_{2}}{M_{1}-M_{2}},
$$

where the quadratic term coefficient $Q$, and the period $P$ are in days. Adopting the masses obtained by $\mathrm{Lu}$ (1992), $M_{1}=1.18 \mathrm{M}_{\odot}$ and $M_{2}=0.69 \mathrm{M}_{\odot}$, the rate of mass transfer $\dot{M}=1.52 \times 10^{-8} \mathrm{M}_{\odot} /$ yr is obtained. Our calculations yield a relatively slower rate than that derived by Chaubey $(1993)\left(3.94 \times 10^{-7} \mathrm{M}_{\odot} / \mathrm{yr}\right)$ by about one order of magnitude.
The light time effect due to the presence of the third body is clearly visible in the upper panel of Fig. 2 after the removal of the parabola. The residuals are also presented in the lower panel of the Fig. 2. The final solution of the orbital parameters are listed in the Table 2. The results show significant differences from those obtained earlier by Vinkó (1992).

\subsubsection{Magnetic Activity}

The quasi-sine variation shown in Fig. 1 may result from cyclic magnetic activity, proposed by Applegate (1992). Magnetic activities seen in low-mass late-type stars may produce this kind of period variation because of their rapid rotation and outer convective layers (Richards \& Albright 1993). Changes of the magnetic field distribution result in changes of angular momentum distribution. Gravitational quadrupole coupling produces changes in the internal structure of the active star which results in a period variation. To compute the amplitude of the period oscillation, one could use the following equation (Rovithis-Livaniou et al. 2000),

$$
\Delta P=A \sqrt{2\left[1-\cos \left(2 \pi P_{e} / P_{3}\right)\right]}
$$

as $\Delta P=2.25 \times 10^{-6}$ with $P_{3}=20081.4$ days. Thus, the rate of period variation is found to be $\Delta P / P=$ $3.161 \times 10^{-6}$. Following Lanza \& Rodonò $(2002)$,

$$
\frac{\Delta P}{P}=-9 \frac{\Delta Q}{M a^{2}}
$$

the variation in the quadrupole moment can be estimated to be $\Delta Q=6.5 \times 10^{49} \mathrm{~g} \cdot \mathrm{cm}^{2}$ for the secondary evolved late type component; where $M$ is the mass of the active star and the separation $a$ between both components can be determined with the Kepler's third low, 


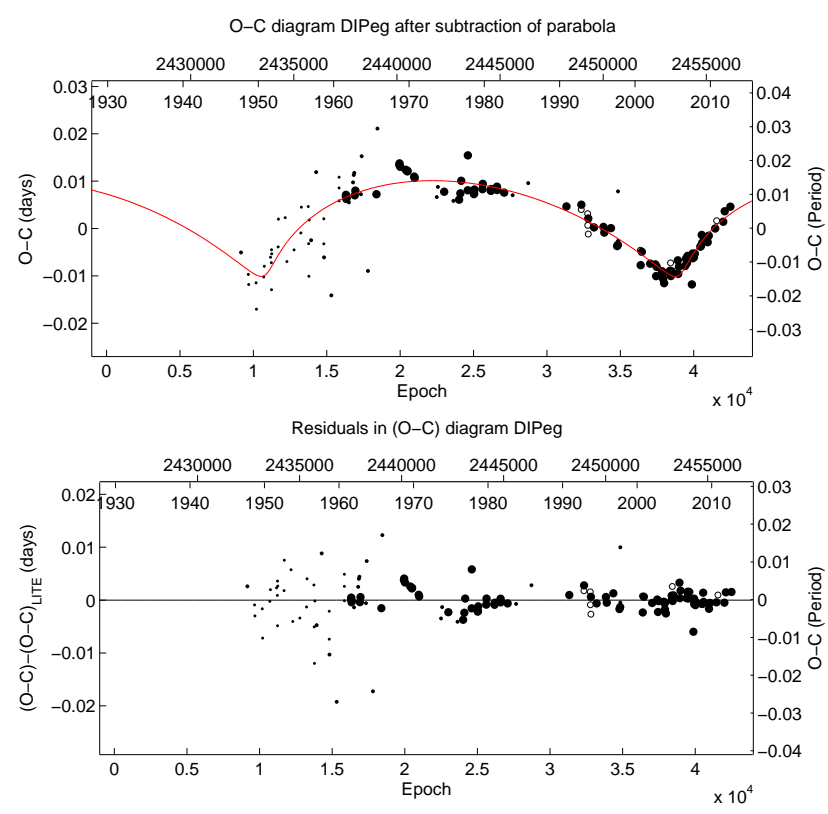

Fig. 2. - LITE solution made after the removal of a parabola (upper panel) and residuals (lower panel) for DI Peg.

$$
a=\left[74.5 \cdot P^{2}\left(M_{1}+M_{2}\right)\right]^{1 / 3} .
$$

Assuming conservation of the orbital angular momentum, Lanza \& Rodonó (1999, 2004) have argued that magnetic variation could be detectable if the quadrupole moment $\Delta Q$ is of the order $10^{51}-$ $10^{52} \mathrm{~g} \mathrm{~cm}^{2}$ for Algol-type binaries, which indicating that the obtained $\Delta Q$ value of the secondary component of DI Peg is not typical value for the close binaries. Therefore, the magnetic activity proposed by Applegate is not a possible mechanism to explain the cyclic variation of DI Peg. In addition, magnetic activity cycle of 55 years is considerably longer than expected in such low mass solar type stars in comparison to our sun.

\section{CONCLUSIONS}

The period variation of the Algol-type semi-detached eclipsing binary DI Peg was discussed using its $O-C$ diagram. Accurate pe and CCD minima collected during the last two decades were analyzed. The available collected times of minima covering about 84 years. We have applied the hypothesis of orbiting the system around the common center of mass with a third unseen companion, so-called light time effect, see Irwin (1959), together with a long term orbital period modualation increase shown, in Fig. 1, as a blue dashed parabolic curve. This approach was used in a programme by Zasche et al. (2009).
A quasi-sinusoidal variation, seen in Fig. 1, has a period of about 55 years, superimposed on a quadratic orbital period increase of rate $d P / d t=3.8 \times 10^{-11}$ $\mathrm{d} /$ cycle $\left(=1.9 \times 10^{-8} \mathrm{~d} / \mathrm{yr}\right)$, corresponding to a time scale of $3.65 \times 10^{7} \mathrm{yr}$. This period increase can be interpreted to be due to mass transfer from the evolved secondary star to its primary more massive companion, which is a common mechanism in such Algol semidetached eclipsing close binary systems.

The existence of the third component in the DI Peg system has been independently proven in three different ways: first, by Rucinski (1967) during his study to obtain the geometrical elements, he introduced an additional third light of about $24 \%$ in order to obtain the geometrical elements; second, by Lu (1992), who observed a direct spectral evidence by using highdispersion spectrograph; he obtained a well determined radial velocity of $+40 \pm 0.3 \mathrm{Km} / \mathrm{s}$ for a third body; finally, through the present work, by studying the $(O-C)$ residual diagram. A well defined light time effect with a period of about 55 years and an amplitude of 0.02 days has been determined.

The Applegate (1992) mechanism was used in testing the probable presence of enough quadrupole momentum which may cause such cyclic variation in the $O-C$ diagram. The result shows that the mechanism of Applegate cannot explain the cyclical period variation of DI Peg.

\section{ACKNOWLEDGMENTS}

This paper has made use of the variable star observations from the AAVSO and the BBSAG International Database contributed by observers worldwide. Many thanks to the NASA Astrophysics Data Service and to the Cracow Eclipsing Binaries Minima Database. Thanks go to Dr. Petr Zasche for the use of his program concerning the determination of the LITE due to the third body.

\section{REFERENCES}

Applegate, J. H. 1992, A Mechanism for Orbital Period Modulation in Close Binaries, ApJ, 385, 621

Awadalla, N., Chochol, D., Hanna, M., \& Pribulla, T. 2004, Orbital Period Study of AK Her, CoSka, 34, 20

Biermann, P., \& Hall, D. 1973, A Model to Explain Alternate Period Changes in Algol-Like Binaries, A\&A, 27, 249

Binnendijk, L. 1973, The Light Variation of the Eclipsing Variable DI Pegasi, AJ, 78, 97

Chou, K. C., \& Kitamura, M. 1968, Analysis on the Photometric Orbit of DI Pegasi, JKAS, 1, 1

Chaubey, U. S. 1982, Photometric Elements and Evolutionary Status of Eclipsing Binary DI Pegasi, Ap\&SS, 81, 283

Chaubey, U. S. 1993, Mass Transfer in Algols and Its Implications to the Mass Transfer Theory, BASI, 21, 597 
Gaposchkin, S. 1952, Variable Stars in Milton Field 22, Harvard Ann., 118, 41

Hanna, M. A. 2006, Study of Period Variation of the Eclipsing Binary System W Delphini, JKAS, 39, 129

Hanna, M. A. 2012, Orbital Period Changes and Possible Stellar Wind Mass Loss in the Algol-Type Binary System AT Pegasi, NRIAG Journal of Astronomy and Geophysics, 1,87

Irwin, J. B. 1959, Standard Light-Time Curves, AJ, 64, 149

Jensch, A. 1934, Ergebnisse Photographischer und Visueller Beobachtungen an 7 Veränderlichen, AN, 252, 393.

Kholopov, P. N., Samus, N. N., Frolov, M. S., and 13 coauthors. 1998, The Combined General Catalog of Variable Stars, 4.1 Edition

Karska, A., \& Maciejewski, G. 2003, CCD Times of Minima of Some Eclipsing Binaries in 2002, IBVS, 5380

Kennedy, H. D. 1982, Period Variations of the Eclipsing System DI Pegasi, PASAu, 4, 406

Kreiner, J. M., \& Ziolkowski, J. 1978, Period Changes and Evolutionary Status of 18 Algol-Type Systems, AcA, 28, 497

Kreiner, J. M., Pajdosz, G., Tremko, J., \& Zola, S. 1994, Investigation of Semidetached Eclipsing Binary RZ Draconis, A\&A, 285, 459

Kreiner, J. M. 2004, Up-To-Date Linear Elements of Eclipsing Binaries, AcA, 54, 207

Lu, W. 1992, A Truly Triple System DI Pegasi, AcA, 42, 73

Mallama, A. D. 1980, New Ephemerides for 120 Eclipsing Binary Stars, ApJS, 44, 241

Lanza, A. F., \& Rodonó, M. 1999, Orbital Period Modulation and Quadrupole Moment Changes in Magnetically Active Close Binaries, A\&A, 349, 887

Lanza, A. F., \& Rodonó, M. 2002, Gravitational QuadrupoleMoment Variations in Active Binaries, AN, 323, 424

Lanza, A. F., \& Rodonò, M. 2004, Magnetic Activity and Dynamics of Close Binaries, AN, 325, 393

Mardirossian, F., Predolin, F., \& Giuricin, G. 1980, Revised Photometric Elements of the Eclipsing Binary DI-Pegasi, A\&A, 91, 254

Morgenroth, O. 1934, 77 Neue Verderliche, AN, 252, 393

Mayer, P. 1990, Eclipsing Binaries with Light-Time Effect, BAICz, 41, 231

Piotrowski, S. L., Rucinski, S. M., \& Semeniuk, I. 1974, Anomalies in Light Curves of Eclipsing Variables, AcA, 24,389

Pribulla, T. 1998, Efficiency of Mass Transfer and Outflow in Close Binaries, CoSka, 28, 101

Qian, S. 2000, Long-Time Behavior of Orbital Periods of Some Algol-Type Eclipsing Binaries, A\&AS, 146, 377

Rafert, J. B. 1982, Periodic Ephemerides for 49 Eclipsing Binary-Star Systems, PASP, 94, 485

Richards, M. T., \& Albright, G. E. 1993, Evidence of Magnetic Activity in Short-Period Algol Binaries, ApJS, 88, 199
Rovithis-Livaniou, H., Kranidiotis, A. N., Rovithis, P., \& Athanassiades, G. 2000, Study of the Period Changes of X Trianguli, A\&A, 354, 904

Rucinski, S. M. 1967, The Eclipsing Binary System DI Pegasi, AcA, 17, 271

Şenavci, H. V., Tanriverdi, T., Torun, E., and 7 coauthors. 2007, Photoelectric Minima of Some Eclipsing Binary Stars, IBVS, 5754

Tremko, J., Kreiner, J. M., \& Pribulla, T. 2004, The Third Body in the System UX Her, CoSka, 34, 33

Vinkó, J. 1992, Photometric Orbit of the Triple System DI Pegasi, IBVS, 3757

von Ahnert, P. 1974, Die Periodenanderungen des Algolsterns DI Peg, MitVS, 6, 158

Wilson, R. E. 1979, Eccentric Orbit Generalization and Simultaneous Solution of Binary Star Light and Velocity Curves, ApJ, 234, 1054

Wolf, M., Kotkova, L., Brat, L., Hanzl, D., Hornoch, K., Lehky, M., Smelcer, L., \& Zasche, P. 2007, CL Aurigae: A Triple System with Mass Transfer, IBVS, 5780

Wood, D. B. 1972, A Computer Program for Modeling Non-Spherical Eclipsing Binary Systems, Goddard Space Flight Center, Greenbelt, Maryland, U.S.A.

Zasche, P, Liakos, A., Wolf, M., \& Niarchos, P. 2008, Period Changes in Six Semi-Detached Algol-Type Binaries, NA, 13, 405

Zasche, P., Liakos, A., Niarchos, P., Wolf, M., Manimanis, V., \& Gazeas, K. 2009, Period Changes in Six Contact Binaries: WZ And, V803 Aql, DF Hya, PY Lyr, FZ Ori, and AH Tau, NA, 14, 121 
APPENDIX A. TIMES OF MINIMA

Table 3.

Photoelectric and CCD times of minima

\begin{tabular}{|c|c|c|c|c|}
\hline J.D Hel. & type & $\overline{\mathrm{wt}}$ & $\overline{O-C}$ & $\overline{\text { Ref. }}$ \\
\hline 32441.4370 & $\mathrm{~V}$ & 1 & -0.0098 & [1] \\
\hline 32794.4970 & $\mathrm{v}$ & 1 & -0.0105 & [1] \\
\hline 32809.4430 & $\mathrm{~V}$ & 1 & -0.0127 & {$[1]$} \\
\hline 33170.3340 & $\mathrm{~V}$ & 1 & -0.0126 & {$[1]$} \\
\hline 33187.4120 & $\mathrm{~V}$ & 1 & -0.0182 & [1] \\
\hline 33538.3440 & $\mathrm{v}$ & 1 & -0.0117 & {$[1]$} \\
\hline 33570.3780 & $\mathrm{v}$ & 1 & -0.0094 & [1] \\
\hline 33871.4780 & $\mathrm{v}$ & 1 & -0.0077 & [1] \\
\hline 33913.4740 & $\mathrm{~V}$ & 1 & -0.0089 & {$[2]$} \\
\hline 33916.3240 & $\mathrm{v}$ & 1 & -0.0062 & {$[2]$} \\
\hline 33918.4510 & $\mathrm{~V}$ & 1 & -0.0146 & [2] \\
\hline 33928.4240 & $\mathrm{v}$ & 1 & -0.0071 & {$[1]$} \\
\hline 34239.4890 & $\mathrm{v}$ & 1 & -0.0058 & [1] \\
\hline 34254.4430 & $\mathrm{~V}$ & 1 & 0.0000 & [1] \\
\hline 34580.4550 & $\mathrm{~V}$ & 1 & 0.0001 & {$[1]$} \\
\hline 34664.4400 & $\mathrm{~V}$ & 1 & -0.0092 & [1] \\
\hline 35010.3850 & $\mathrm{~V}$ & 1 & -0.0070 & [1] \\
\hline 35341.3830 & $\mathrm{~V}$ & 1 & -0.0036 & {$[1]$} \\
\hline 35366.3020 & $\mathrm{~V}$ & 1 & 0.0018 & [1] \\
\hline 35699.4320 & $\mathrm{~V}$ & 1 & 0.0017 & [1] \\
\hline 35719.3550 & $\mathrm{~V}$ & 1 & -0.0061 & {$[1]$} \\
\hline 35731.4490 & $\mathrm{~V}$ & 1 & -0.0130 & [1] \\
\hline 35746.4090 & $\mathrm{v}$ & 1 & -0.0012 & [1] \\
\hline 35838.2290 & pg & 3 & -0.0055 & {$[3]$} \\
\hline 36079.5490 & pg & 3 & 0.0087 & [3] \\
\hline 36450.3900 & $\mathrm{~V}$ & 1 & -0.0066 & {$[1]$} \\
\hline 36455.3780 & $\mathrm{~V}$ & 1 & -0.0013 & [1] \\
\hline 36462.4880 & $\mathrm{pg}$ & 3 & -0.0095 & [3] \\
\hline 36818.3880 & pg & 3 & -0.0177 & [3] \\
\hline 37193.5350 & vis & 1 & 0.0021 & [1] \\
\hline 37193.5400 & $\mathrm{~V}$ & 1 & 0.0071 & [1] \\
\hline 37196.3850 & $\mathrm{v}$ & 1 & 0.0008 & [1] \\
\hline 37522.3946 & pe & 10 & 0.0025 & {$[4]$} \\
\hline 37523.4620 & pe & 10 & 0.0022 & {$[4]$} \\
\hline 37527.3776 & pe & 10 & 0.0028 & {$[4]$} \\
\hline 37544.4610 & pe & 10 & 0.0026 & {$[4]$} \\
\hline 37559.4093 & pe & 10 & 0.0027 & {$[4]$} \\
\hline 37668.3160 & $\mathrm{pg}$ & 3 & 0.0015 & [3] \\
\hline 37870.4760 & pg & 3 & 0.0057 & [3] \\
\hline 37907.4922 & pg & 3 & 0.0074 & [3] \\
\hline 37932.4060 & pg & 3 & 0.0076 & [3] \\
\hline 37956.6032 & pe & 10 & 0.0031 & [1] \\
\hline 37983.6528 & pe & 10 & 0.0037 & [1] \\
\hline 38255.5660 & pg & 3 & 0.0030 & {$[3]$} \\
\hline 38290.4530 & pg & 3 & 0.0110 & [3] \\
\hline 38591.5270 & $\mathrm{pg}$ & 3 & -0.0134 & [3] \\
\hline 39006.5324 & pe & 10 & 0.0031 & {$[4]$} \\
\hline 39056.3730 & pg & 3 & 0.0165 & [3] \\
\hline 40114.8356 & pe & 10 & 0.0081 & {$[5]$} \\
\hline 40127.6488 & pe & 10 & 0.0086 & [5] \\
\hline 40159.6796 & pe & 10 & 0.0077 & [5] \\
\hline 40424.4746 & pe & 10 & 0.0070 & [6] \\
\hline 40500.6394 & pe & 10 & 0.0074 & {$[5]$} \\
\hline 40512.7402 & pe & 10 & 0.0073 & [5] \\
\hline 40837.3269 & pe & 10 & 0.0058 & [7] \\
\hline
\end{tabular}

Table 3. - continued

\begin{tabular}{|c|c|c|c|c|}
\hline J.D Hel. & type & $\mathrm{wt}$ & $O-C$ & Ref. \\
\hline 40859.3930 & pe & 10 & 0.0056 & 7 \\
\hline 41928.5370 & pg & 3 & 0.0013 & [8] \\
\hline 41983.3490 & pg & 3 & 0.0034 & [9] \\
\hline 42289.4285 & pe $B \mathrm{~V}$ & 10 & 0.0019 & [10] \\
\hline 42739.2950 & pg & 3 & 0.0004 & {$[11]$} \\
\hline 43015.4802 & pe & 10 & 0.0008 & {$[12]$} \\
\hline 43071.0029 & pe & 10 & 0.0019 & {$[13]$} \\
\hline 43112.2910 & pe & 10 & 0.0046 & [14] \\
\hline 43434.0295 & pe & 10 & 0.0021 & [13] \\
\hline 43725.5179 & pe $\mathrm{V}$ & 10 & 0.0017 & {$[15]$} \\
\hline 43729.4334 & pe B V & 10 & 0.0022 & [15] \\
\hline 43756.48315 & pe B V & 10 & 0.0029 & [15] \\
\hline 43780.3277 & pe $\mathrm{V}$ & 10 & 0.0016 & {$[15]$} \\
\hline 44143.35645 & pe B V & 10 & 0.0040 & [16] \\
\hline 44144.42295 & pe B V & 10 & 0.0028 & [16] \\
\hline 44164.3545 & pe & 10 & 0.0035 & {$[17]$} \\
\hline 44219.1650 & pe & 10 & 0.0041 & {$[17]$} \\
\hline 44502.4654 & pe & 10 & 0.0016 & [18] \\
\hline 44543.0401 & pe & 10 & 0.0027 & {$[13]$} \\
\hline 44557.9879 & pe & 10 & 0.0024 & [13] \\
\hline 44567.2420 & pe & 10 & 0.0029 & [19] \\
\hline 44843.4272 & pe & 10 & 0.0033 & {$[20]$} \\
\hline 44848.4102 & pe & 10 & 0.0036 & {$[20]$} \\
\hline 44853.3920 & pe & 10 & 0.0027 & [21] \\
\hline 45196.4870 & pe & 10 & 0.0022 & {$[22]$} \\
\hline 45609.3400 & pg & 3 & 0.0017 & {$[23]$} \\
\hline 46360.3090 & $\mathrm{v}$ & 1 & 0.0057 & {$[24]$} \\
\hline 48219.5690 & pe & 10 & -0.0001 & {$[25]$} \\
\hline 48935.3002 & pe & 10 & -0.0003 & {$[26]$} \\
\hline 48939.2161 & pe & 10 & 0.0006 & {$[26]$} \\
\hline 49246.3631 & pe & 10 & -0.0012 & {$[26]$} \\
\hline 49248.4963 & pe & 10 & -0.0034 & {$[26]$} \\
\hline 49276.2546 & pe & 10 & -0.0060 & {$[26]$} \\
\hline 49277.3259 & pe & 10 & -0.0024 & {$[26]$} \\
\hline 49553.5085 & pe & 10 & -0.0046 & {$[26]$} \\
\hline 50008.3599 & pe & 10 & -0.0039 & {$[27]$} \\
\hline 50008.3603 & $\operatorname{ccd}$ & 10 & -0.0035 & [28] \\
\hline 50050.3564 & pe & 10 & -0.0045 & {$[26]$} \\
\hline 50376.3686 & pe & 10 & -0.0042 & [29] \\
\hline 50672.4811 & pe & 10 & -0.0074 & {$[30]$} \\
\hline 50712.3428 & pe & 10 & -0.0074 & {$[31]$} \\
\hline 50717.3370 & $\mathrm{pg}$ & 3 & 0.0041 & {$[32]$} \\
\hline 50719.4620 & pg & 3 & -0.0066 & {$[33]$} \\
\hline 51807.4721 & pe & 10 & -0.0076 & {$[34]$} \\
\hline 51818.5020 & pe & 10 & -0.0109 & {$[34]$} \\
\hline 51868.3321 & pe & 10 & -0.0079 & {$[34]$} \\
\hline 52278.3363 & pe & 10 & -0.0100 & {$[34]$} \\
\hline 52530.3191 & pe & 10 & -0.0102 & {$[35]$} \\
\hline 52567.3312 & pe & 10 & -0.0126 & {$[35]$} \\
\hline 52594.3820 & pe & 10 & -0.0108 & {$[36]$} \\
\hline 52843.5166 & pe & 10 & -0.0119 & {$[37]$} \\
\hline 52888.3606 & pe & 10 & -0.0124 & [38] \\
\hline 52903.3083 & pe & 10 & -0.0128 & {$[39]$} \\
\hline 52908.2924 & pe & 10 & -0.0114 & [39] \\
\hline 52950.2871 & pe & 10 & -0.0139 & {$[39]$} \\
\hline 53236.4400 & pe & 10 & -0.0112 & {$[40]$} \\
\hline
\end{tabular}


Table 3. - continued

\begin{tabular}{lcccc}
\hline J.D Hel. & type & wt & $O-C$ & Ref. \\
\hline 53262.4225 & pe & 10 & -0.0100 & {$[41]$} \\
53265.6239 & ccd & 10 & -0.0118 & {$[42]$} \\
53267.7591 & ccd & 10 & -0.0120 & {$[42]$} \\
53272.7415 & ccd & 10 & -0.0123 & {$[42]$} \\
53282.7067 & ccd & 10 & -0.0125 & {$[42]$} \\
53325.4174 & pe & 10 & -0.0108 & {$[41]$} \\
53614.4169 & ccd & 10 & -0.0088 & {$[43]$} \\
53634.3450 & ccd & 10 & -0.0116 & {$[44]$} \\
53671.36089 & ccd C & 10 & -0.0101 & {$[45]$} \\
53967.4772 & ccd & 10 & -0.0094 & {$[46]$} \\
53991.3226 & ccd & 10 & -0.0099 & {$[47]$} \\
54024.4239 & ccd & 10 & -0.0080 & {$[48]$} \\
54027.27059 & ccd R & 10 & -0.0086 & {$[45]$} \\
54059.3020 & pe B V & 10 & -0.0090 & {$[49]$} \\
54096.31773 & ccd R & 10 & -0.0077 & {$[45]$} \\
54335.4878 & pe & 10 & -0.0079 & {$[50]$} \\
54335.48869 & ccd R V I & 10 & -0.0070 & {$[45]$} \\
54394.5693 & ccd & 10 & -0.0072 & {$[51]$} \\
54416.6361 & ccd & 10 & -0.0067 & {$[51]$} \\
54436.5670 & ccd & 10 & -0.0067 & {$[52]$} \\
54710.6180 & ccd & 10 & -0.0050 & {$[53]$} \\
54738.3787 & ccd V & 10 & -0.0051 & {$[54]$} \\
54799.5955 & ccd & 10 & -0.0045 & {$[55]$} \\
54774.6840 & ccd & 10 & -0.0024 & {$[56]$} \\
55044.4623 & ccd V R & 10 & -0.0029 & {$[57]$} \\
55064.3920 & pe V & 10 & -0.0037 & {$[58]$} \\
55064.3929 & pe V & 10 & -0.0028 & {$[58]$} \\
55085.7474 & ccd & 10 & -0.0028 & {$[59]$} \\
55116.3557 & ccd C & 10 & -0.0026 & {$[57]$} \\
55429.5569 & ccd V & 10 & -0.0006 & {$[60]$} \\
55498.2485 & ccd R & 10 & 0.0007 & {$[61]$} \\
55561.2439 & ccd V R & 10 & 0.0004 & {$[62]$} \\
55820.3461 & pe & 10 & 0.0013 & {$[63]$} \\
55887.2592 & pe & 10 & 0.0037 & {$[63]$} \\
56163.4447 & ccd & 10 & 0.0044 & {$[64]$} \\
\hline & & & &
\end{tabular}

Ref. [1] cf, Chou, K. \& Kitamura, M. (1968), JKAS 1, 1; [2] Kruszewski, A. (1956), AcA 6, 140; [3] Huth, H. (1966), Sonneberg 3,170; [4] Rucinski, S.M. (1967), AcA 17, 271; [5] Binnendijk, L. (1973), AJ. 78, 97; [6] IBVS 456; [7] IBVS 530; [8] AN 298, 121; [9] IBVS 978; [10] IBVS 1053; [11] AN 300, 165; [12] IBVS 1358; [13] IBVS 2118; [14] BBSAG Bull. 31; [15] IBVS 1495; [16] IBVS 1908; [17] Chaubey, U.S. (1982), Ap\&SS 81, 283; [18] BBSAG Bull. 54; [19] BBSAG Bull. 51; [20] IBVS 2159; [21] BRNO 26; [22] IBVS 2385; [23] MVS 10, 104; [24] BAV-M 46; [25] AAVSO 2; [26] IBVS 4380; [27] IBVS 4382; [28] BBSAG Bull. 110; [29] IBVS 4562; [30] IBVS 4534; [31] IBVS 4562; [32] BAV-M 113; [33] IBVS 4606; [34] IBVS 5296; [35] IBVS 5484; [36] IBVS 5407; [37] IBVS 5791; [38] IBVS 5592; [39] IBVS 5643; [40] IBVS 5649; [41] IBVS 5657; [42] IBVS 5843; [43] IBVS 5662; [44] IBVS 5731; [45] Oejv 74; [46] IBVS 5777; [47] IBVS 5746; [48] IBVS 5761; [49] IBVS 5754; [50] IBVS 5801; [51] JAVSO 36, 171; [52] IBVS 5814; [53] JAVSO 36, 186; [54] IBVS 5898; [55] JAVSO 37,
44; [56] IBVS 5871; [57] IBVS 5924; [58] IBVS 5941; [59] JAVSO 38, 183; [60] IBVS 5988; [61] IBVS 5980; [62] BRNO 37; [63] IBVS 6026; [64] IBVS 6044; 
Table 4

Unused Data (see the text)

\begin{tabular}{|c|c|c|c|c|c|c|c|c|c|c|c|}
\hline "J.D Hel. & $\overline{\text { Ref. }}$ & "J.D Hel. & $\overline{\overline{\text { Ref. }}}$ & "J.D Hel. & $\overline{\overline{\text { Ref. }}}$ & "J.D Hel. & $\overline{\overline{\text { Ref. }}}$ & J.D Hel. & $\overline{\overline{\text { Ref. }}}$ & J.D Hel. & $\overline{\overline{\text { Ref. }}}$ \\
\hline 25644.315 & [1] & 42304.376 & \begin{tabular}{|l|}
16 \\
\end{tabular} & 44134.458 & $35]$ & 44883.283 & \begin{tabular}{|l|}
46 \\
\end{tabular} & 46290.538 & $52]$ & 48205.336 & 59 \\
\hline 27738.474 & {$[1]$} & 42403.317 & {$[17]$} & 44164.3554 & {$[36]$} & 44890.41 & {$[46]$} & 46290.545 & {$[52]$} & 48266.552 & {$[22]$} \\
\hline 28452.421 & [1] & 42403.322 & {$[17]$} & 44166.492 & {$[36]$} & 44893.255 & $46]$ & 46305.501 & {$[52]$} & 48480.814 & {$[22]$} \\
\hline 31273.346 & {$[1]$} & 42403.324 & {$[17]$} & 44189.267 & {$[37]$} & 44900.387 & {$[46]$} & 46320.45 & {$[52]$} & 48481.524 & 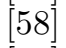 \\
\hline 38399.362 & {$[2]$} & 42739.3 & {$[18]$} & 44435.565 & {$[38]$} & 44910.33 & {$[46]$} & 46344.65 & $22]$ & 48506.423 & 58 \\
\hline 39024.412 & {$[3]$} & 42754.247 & [19] & 44440.54 & {$[38]$} & 44925.284 & {$[46]$} & 46350.345 & {$[53]$} & 48545.587 & {$[22]$} \\
\hline 39026.463 & [2] & 42776.296 & [19] & 44445.525 & [38] & 45170.858 & [22] & 46355.324 & [54] & 48859.497 & [58] \\
\hline 39046.394 & {$[2]$} & 42786.271 & {$[20]$} & 44455.483 & {$[39]$} & 45201.469 & {$[45]$} & 46360.304 & $55]$ & 48859.5 & {$[58]$} \\
\hline 39056.361 & {$[2]$} & 42786.275 & {$[20]$} & 44455.489 & {$[39]$} & 45201.47 & {$[45]$} & 46382.371 & $55]$ & 48859.507 & {$[58]$} \\
\hline 39056.364 & {$[2]$} & 42796.24 & [20] & 44455.49 & {$[39]$} & 45201.472 & {$[47]$} & 46413.698 & $22]$ & 48859.512 & -0 \\
\hline 39062.346 & {$[2]$} & 42990.57 & [21] & 44455.491 & {$[39]$} & 45228.52 & {$[45]$} & 46422.238 & $53]$ & 48863.766 & {$[22]$} \\
\hline 39352.479 & {$[4]$} & 42993.412 & {$[21]$} & 44455.497 & {$[39]$} & 45228.523 & {$[45]$} & 46656.423 & {$[56]$} & 48873.733 & {$[22]$} \\
\hline 39387.36 & [4] & 43013.351 & [21] & 44474.703 & [22] & 45231.369 & [48] & 46656.424 & [56] & 49215.413 & [58] \\
\hline 39389.496 & {$[4]$} & 43034.701 & {$[22]$} & 44490.364 & {$[40]$} & 45235.645 & $22]$ & 46678.485 & {$[57]$} & 49224.65 & {$[22]$} \\
\hline 39407.289 & {$[4]$} & 43040.398 & {$[23]$} & 44490.366 & {$[40]$} & 45258.408 & {$[45]$} & 46678.487 & {$[57]$} & 49241.735 & {$[22]$} \\
\hline 40128.36 & {$[5]$} & 43069.57 & {$[22]$} & 44497.486 & {$[41]$} & 45258.418 & {$[45]$} & 46678.489 & {$[57]$} & 49333.56 & ] \\
\hline 40175.343 & {$[4]$} & 43069.583 & {$[22]$} & 44502.469 & {$[40]$} & 45258.422 & {$[45]$} & 46678.49 & $53]$ & 49543.544 & $63]$ \\
\hline 40471.454 & {$[6]$} & 43134.36 & {$[24]$} & 44512.434 & {$[40]$} & 45554.521 & {$[45]$} & 46738.276 & {$[57]$} & 49543.55 & {$[66]$} \\
\hline 40476.437 & [6] & 43154.288 & [25] & 44517.416 & {$[41]$} & 45554.522 & {$[45]$} & 46743.273 & [58] & 49602.63 & {$[22]$} \\
\hline 41155.502 & {$[7]$} & 43311.594 & {$[26]$} & 44517.419 & $42]$ & 45554.527 & {$[45]$} & 46759.639 & $22]$ & 50396.3 & $65]$ \\
\hline 41155.506 & {$[7]$} & 43371.387 & {$[27]$} & 44524.534 & {$[41]$} & 45579.447 & {$[45]$} & 46769.607 & $22]$ & 50423.356 & {$[65]$} \\
\hline 3.556 & [8] & 43391.319 & [28] & 44532.364 & {$[43]$} & 45609.344 & {$[45]$} & 46774.591 & {$[22]$} & 50672.4793 & \\
\hline 41550.562 & [9] & 43393.457 & [28] & 44567.245 & {$[41]$} & 45621.445 & {$[45]$} & 46779.564 & $22]$ & 50754.348 & {$[67]$} \\
\hline 41563.381 & [9] & 43433.323 & [29] & 44593.584 & {$[22]$} & 45624.292 & [49] & 46999.52 & [59] & 52542.7862 & {$[68]$} \\
\hline 41565.512 & [9] & 43435.461 & {$[29]$} & 44636.287 & {$[42]$} & 45671.275 & {$[45]$} & 47014.466 & $60]$ & 52848.5081 & {$[69]$} \\
\hline 41580.46 & [9] & 43495.244 & {$[30]$} & 44823.49 & {$[44]$} & 45915.423 & {$[50]$} & 47054.333 & [59] & 52572.6843 & {$[68]$} \\
\hline 41595.407 & {$[10]$} & 43517.318 & {$[30]$} & 44823.493 & {$[44]$} & 45976.643 & {$[22]$} & 47066.429 & {$[59]$} & 52573.0329 & {$[68]$} \\
\hline 41605.373 & {$[10]$} & 43689.571 & {$[31]$} & 44823.494 & {$[45]$} & 45976.65 & {$[22]$} & 47091.346 & {$[59]$} & 52848.5024 & {$[69]$} \\
\hline 41605.378 & [10] & 43776.414 & [29] & 44823.495 & {$[45]$} & 45981.629 & {$[22]$} & 47107.718 & {$[22]$} & 53236.44 & {$[69]$} \\
\hline 41657.337 & [11] & 43791.354 & {$[32]$} & 44823.496 & {$[45]$} & 45992.303 & {$[51]$} & 47387.461 & 61] & 53236.4476 & {$[69]$} \\
\hline 41928.537 & {$[3]$} & 43791.37 & {$[32]$} & 44823.497 & {$[45]$} & 46002.261 & {$[51]$} & 47464.344 & {$[22]$} & 53251.381 & [70] \\
\hline 41931.375 & {$[12]$} & 43802.76 & {$[22]$} & 44823.498 & {$[45]$} & 46019.349 & {$[52]$} & 47469.315 & {$[22]$} & 53619.3969 & {$[69]$} \\
\hline 41941.353 & {$[12]$} & 43803.465 & {$[32]$} & 44823.5 & {$[45]$} & 46028.609 & {$[22]$} & 47474.318 & $62]$ & 54070.3254 & [71] \\
\hline 41988.321 & {$[13]$} & 43806.309 & {$[32]$} & 44823.503 & {$[45]$} & 46028.611 & {$[22]$} & 47794.62 & $22]$ & 54298.468 & to \\
\hline 42008.263 & {$[13]$} & 43863.256 & {$[33]$} & 44853.392 & {$[45]$} & 46029.316 & {$[51]$} & 47851.561 & {$[22]$} & & \\
\hline 42274.486 & {$[14]$} & 43878.202 & {$[33]$} & 44853.392 & {$[46]$} & 46033.585 & {$[22]$} & 47853.693 & [22] & & \\
\hline 42289.427 & {$[15]$} & 44092.46 & {$[34]$} & 44853.395 & {$[45]$} & 46038.567 & {$[22]$} & 48123.473 & [58] & & \\
\hline 42301.54 & {$[14]$} & 44117.369 & {$[34]$} & 44853.396 & {$[45]$} & 46038.568 & {$[22]$} & 48123.479 & [58] & & \\
\hline 42304.396 & [14] & 44117.377 & {$[34]$} & 44853.398 & {$[45]$} & 46043.553 & {$[22]$} & 48148.395 & [59] & & \\
\hline
\end{tabular}

[1] cf, Chou, K. \& Kitamura, M. (1968), JKAS 1, 1; [2] Braune, W.\& Hubscher, J. (1967), AN 290, 105; [3] Braune, W. et al., (1977), AN 298, 121; [4] Braune, J. et al. (1970), AN 292, 185; [5] IBVS 328; [6] Prerov, F.H. (1970), CoBrno 9; [7] IBVS 584; [8] BBSAG Bull. 4; [9] BBSAG Bull. 5; [10] BBSAG Bull. 6; [11] BBSAG Bull. 7; [12] BBSAG Bull. 11; [13] BBSAG Bull. 12; [14] Jiri, H. (1976), CoBrno 20; [15] BBSAG Bull. 17; [16] IBVS 1053; [17] BBSAG Bull. 19; [18] BBSAG Bull. 24; [19] BBSAG Bull. 25; [20] BBSAG Bull. 26; [21] BBSAG Bull. 29; [22] AAVSO 2.; [23] BBSAG Bull. 30; [24] BBSAG Bull. 31; [25] BBSAG Bull. 32; [26] BBSAG Bull. 33; [27] BBSAG Bull. 34; [28] BBSAG Bull. 35; [29] Braune, W. et al. (1981), AN 302, 53; [30] BBSAG Bull. 36; [31] BBSAG Bull. 37; [32] BBSAG Bull. 39; [33] BBSAG Bull. 41; [34] BBSAG Bull. 44; [35] BBSAG Bull. 45; [36] BAA 59, 16; [37] BBSAG Bull. 45; [38] BBSAG Bull. 49; [39] MAV 9, 18; [40] BBSAG Bull. 50; [41] BBSAG Bull. 51; [42] BBSAG Bull. 52; [43] BAV-M 32; [44] MVS 9, 89; [45] BRNO 26; [46] BBSAG Bull. 57; [47] BBSAG Bull. 62; [48] BBSAG Bull. 63; [49] BBSAG Bull. 69; [50] BBSAG Bull. 73; [51] BBSAG Bull. 74; [52] BRNO 27; [53] BBSAG Bull. 81; [54] BAv-M 43; [55] MvS 11, 19; [56] BAV-M 46; [57] CoBrno 28; [58] BRNO 31; [59] BBSAG Bull. 86; [60] BAV-M 50; [61] BAV-M 52; [62] BBSAG Bull. 90; [63] BBSAG Bull. 108; [64] BBSAG Bull. 107; [65] BAV-M 101; [66] IBVS 4606; [67] BAV-M 113; [68] Karska, A.\& Maciejewski, G. (2003), IBVS 5380; [69] Lubos Bràt, et al. (2007), Oejv 74; [70] Hubscher, J. et al. (2008), PAV-M 202; [71] Şenavci, H. V. et al. (2007), IBVS 5754. 